\title{
Welding Flow of Low Density Polyethylene Melt
}

\author{
MITSUHASHI Masanori*, NISHIMURA Keiichi*, \\ YAMAMOTO Takehiro**, MORI Noriyasu** and NAKAMURA Kiyoji**
}

\author{
*Nara Prefectural Industrial Research Institute, 129-1 Kashiwagi, Nara, Nara, 630-8031 Japan \\ **Graduate School of Engineering, Osaka University, 2-1 Yamada-Oka, Suita, Osaka, 565-0871 Japan
}

Based on Journal of the Textile Machinery Society of Japan, vol.54, No.11,T165-T173(2001-11)

\begin{abstract}
Weld-lines degrade the mechanical and optical properties of products. This is because the polymer molecules near the weld-line highly orient owing to the elongational flow and the molecular orientation is fixed by solidification of the polymer melts before it returns to a random condition. Birefringence experiments were, therefore, carried out for welding flows of a low density polyethylene (LDPE) past a spider supporting a mandrel. The die used in the present study has a glass window to observe the birefringence pattern. The birefringence in the polymer melts was measured by the photoelastic method. The purpose of the present study is to elucidate the effect of the die temperature, spider shapes and a viscoelastic property of polymer melts on the anisotropy of the molecular orientation in the stress relaxation process downstream of the spider. The birefringence pattern in the welding flow of LDPE was measured at the die temperature $T_{d}=190,20{ }^{\circ} \mathrm{C}$ and the polymer melts temperature $T_{p}=190^{\circ} \mathrm{C}$ for flow rates of $0.7,1.0,2.2,2.9,4.6 \mathrm{~cm}^{3} / \mathrm{s}$. The results showed that complete relaxation of birefringence depended on the flow rate. For high die temperature $\left(205^{\circ} \mathrm{C}\right)$, the distance necessary for the relaxation of molecular orientation was short. Moreover, the birefringence pattern in the welding flow was numerically calculated using the single-mode Giesekus model as a constitutive equation, and the results were compared with the experimental data. The numerical predictions agreed with the experimental results.
\end{abstract}

Keywords: weld-line, birefringence, polymer melts, molecular orientation, Giesekus model

\section{Introduction}

In both the extrusion and the blow molding, weld-lines occur in welding flows that appear when polymer melts pass through a die having a spider. The weld-lines cause the defectiveness in appearance of products and the degradation of their mechanical strength. When flows merge to form a weld surface, a polymer melt is stretched in the flow direction by the elongational flow; hence polymer molecules orient in the flow direction. If the orientation does not relax to a perfect random condition in the diffusive process downstream of the welding region, the above defects appear.

We numerically analyzed viscoelastic welding flows caused by a spider that supported a mandrel under the condition of incompressible isothermal creeping flow [1]. In this analysis, the single mode Giesekus model [2] was used as a constitutive equation. The computational results indicated that the elongation rate and stress near the spider rear end were decreased by a simple modification of spider-rear-end angle. This modification is simpler than the improvement using complicated spider shape proposed by Huang et al. [3] However, the molecular orientation at the exit does not much depend on the spider shape. Moreover it does not reach a perfect random condition in the computational domain at high Weissenberg number when the inlet velocity is large. This tendency strongly appears especially for a fluid having a highly stretch-thickening elongational viscosity. We also numerically analyzed non-isothermal viscoelastic flows to study the effect of temperature, which is an important factor in the polymer processing [4]. In this study, we investigated the relaxation of molecular orientation in the weld-line region when the temperature of channel wall is higher than that of a polymer melt. The computation predicted that both the overshoot of the velocity in the flow direction and the progress of the relaxation at high velocity were caused by the increase in the elongation rate due to the rise in temperature on the channel wall. These results indicate the possibility of improvement in the mechanical strength and the optical property of products by suitable control of the temperature according to the rheological properties of polymer melts to advance the relaxation of polymer orientation.

In experimental studies, the measurement of birefringence has been applied for investigating the molecular orientation in the weld-line region. There are many reports on viscoelastic flow birefringence phenomenon [5-9], while not many studies treat welding flows. Baaijens et al. [10] studied viscoelastic flows past a cylinder by both flow birefringence experiments using low density polyethylene melts and a numerical simulation. They compared the results of the experiment and the simulation. Wei et al. [11] studied the flow birefringence in welding flows using a split die. They realized the welding flow using a thin plate with a quadrilateral cross section. In their paper, the effect of

*Corresponding author Fax:+81-742-34-6705, E-mail: mituhasi@niit.pref.nara.jp 


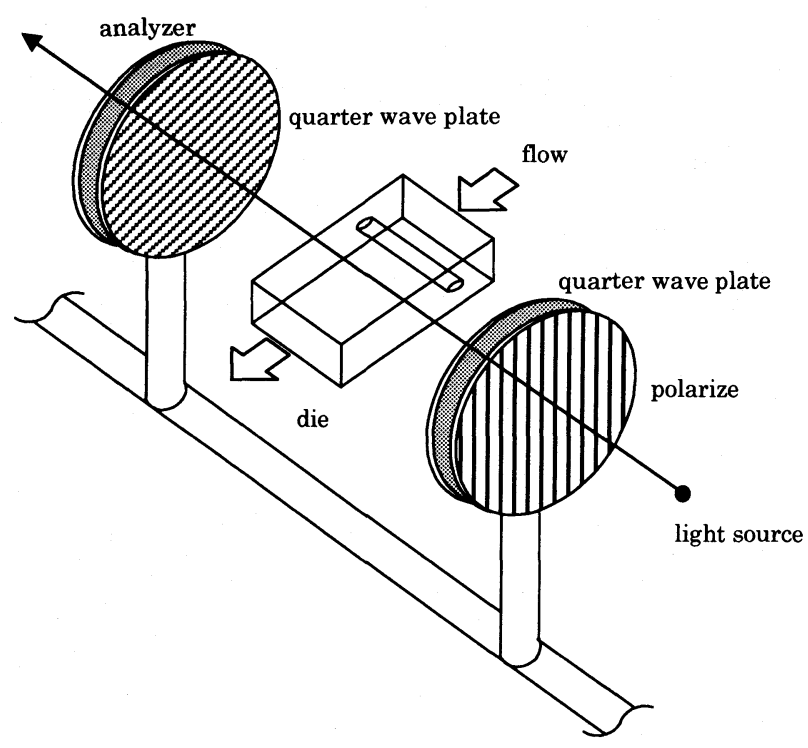

Fig.1 Placement of polarizer, analyzer, and quarter wave plate for birefringence measurements.

neither shape nor temperature was studied. Saito et al. [12] reported the mechanism of the occurrence of the weld around a pin. In this report, they treated unsteady flows having the skin layer, which occur in the injection molding; flows in the extrusion are steady. To the authors' knowledge, there is no report that treats the photoelastic phenomenon in the weld flow in the extrusion processing or the blow molding.

In the present study, we carried out the flow birefringence measurement in the welding flow of a LDPE melt caused by a spider. We investigated the effects of rhelogical properties of the melt, the spider shape, the flow rate, and the die temperature on the relaxation of molecular orientation that appear in the welding region. In addition, we numerically analyzed non-isothermal steady viscoelastic welding flows using the single mode Giesekus model and compared the numerical predictions with the experimental results.

\section{Experiment}

\subsection{Measurement principle}

The observation of stress field in polymer melts in the merging flow past a spider is one method for investigation of the direction and degree of molecular orientation and the relaxation process of the orientation in the region from the spider rear end, where the elongation flow is dominant, to the exit. We observed the flow birefringence phenomena [13-15] using the photoelastic method to measure the growth and relaxation processes of birefringence in the weld-line region and investigated the molecular orientation in that region.

Figure 1 illustrates the placement of a polarizer, an analyzer, and quarter wave planes in the experimental setup for the birefringence measurements. The polarizer and the

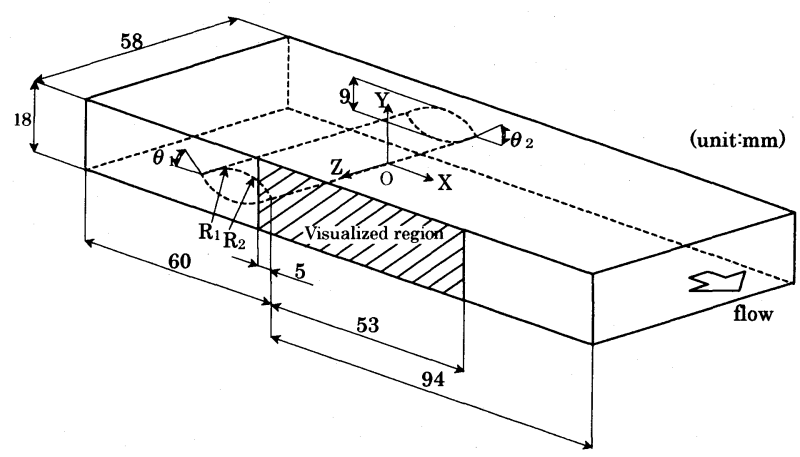

Fig.2 Schematic diagram of a parallel channel with a spider and the coordinate system.

analyzer are placed as their vibration planes are perpendicular to each other. Two quarter-wave planes are inserted between the polarizer and a die to make the circularly polarized light. In this case, the light strength $I$ is

$$
I=I_{0} \sin ^{2} \frac{\delta}{2},
$$

where $\delta$ is the phase shift caused by the flow of polymer melts and $I_{0}$ is the strength of incident light. The birefringence $\Delta n$ is expressed by

$$
\Delta n=k \lambda_{0} / W,
$$

where $k$ is the number of stripe of isocolor line, $\lambda_{0}$ the wave length of the incident light, and $W$ the thickness of the die channel.

\subsection{Experimental setup and method}

Figure 2 shows the schematic diagram of the die channel used for the measurement of the birefringence. The visualized region where a glass plate is inserted is also indicated in the figure. The cross section of the channel is rectilinear and the spider lies at the center of the channel width. The coordinate system is defined as follows: The origin is on the spider-rear-end, the flow direction is $x$, and the $y$ and the $z$ axes are set as shown in this figure.

Figure 3 shows the experimental setup for the measurement of the birefringence. The setup is consists of a twin screw extruder, a glass inserted die for flow visualization, a measurement device of photoelasticity, a video camera, and a computer. The die was heated by a plate heater for the prevention of the radiation. The temperature of the test fluid was measured just upstream of the die entrance. The light source is a mercury lamp: The light passes through a green filter and is translated to a monochromatic light whose wavelength $\lambda_{0}$ is $546 \mathrm{~nm}$. We recorded the images of the birefringence with the video camera, captured them into the computer for image analysis, and computed the birefringence $\Delta n$.

In the present study, we carried out the experiments at five flow rates $Q$ such as $0.7,1.0,2.2,2.9$, and $4.6 \mathrm{~cm}^{3} / \mathrm{s}$. The spider-front-end angle $\theta_{1}$ is $45^{\circ}\left(R_{1}=9.7 \mathrm{~mm}\right)$, and two 


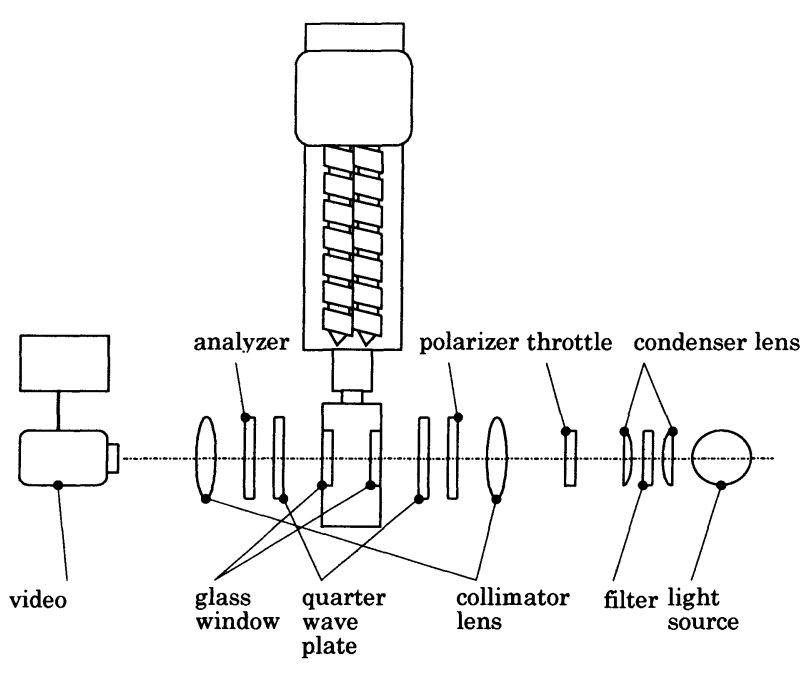

Fig.3 Experimental setup.

spider-rear-end angles $\theta_{2}=45^{\circ}\left(R_{2}=9.7 \mathrm{~mm}\right)$ and $60^{\circ}\left(R_{2}=5.0\right.$ $\mathrm{mm}$ ) were considered (see Fig.6). We used a commercial low density polyethylene melt (LDPE) as a test fluid. The fluid temperature was $190{ }^{\circ} \mathrm{C}$ and the die temperature was 190 or $205^{\circ} \mathrm{C}$. The steady shear viscosity $\eta$ and the first normal stress difference $N_{1}$ of the LDPE at $190^{\circ} \mathrm{C}$ are shown in Fig. 4 together with the prediction of the Giesekus model.

\section{Numerical Method}

In the present study, we computed incompressible non-isothermal viscoelastic creeping flows in the two-dimensional steady state. The single mode Giesekus model was applied as a constitutive equation. The equations of continuity and motion and the constitutive equation were simultaneously solved using the Newton-Raphson method for iteration. The incremental method was applied for the increment of $W e[3,4]$. The shift factor $a_{T}$ for describing the temperature dependence of both the relaxation time and the viscosity was expressed by the WLF equation:

$$
\ln a_{T}=\frac{-c_{1}\left(T-T_{r e f}\right)}{c_{2}+T-T_{r e f}},
$$

where $c_{1}$ and $c_{2}$ are constants, $T$ the temperature, $T_{\text {ref }}$ the reference temperature. The birefringence $\Delta n$ was calculated by the following equation based on the photoelastic law:

$$
\Delta n=C\left(4 \tau_{x y}^{2}+N_{1}^{2}\right)^{1 / 2} \text {, }
$$

where $C$ is the photoelastic coefficient, $\tau_{x y}$ the shear stress, and $N_{1}$ the first normal stress difference.

The parameters of the Giesekus model at $T_{r e f}=190^{\circ} \mathrm{C}$ used in the present computation are as follows: The nonlinear parameter $\alpha$ is 0.15 , the relaxation time $\lambda$ is $4.0 \mathrm{~s}$, the solvent contribution to the viscosity $\eta_{S}$ is $500 \mathrm{~Pa} \cdot \mathrm{s}$, and the polymer contribution to the viscosity $\eta_{P}$ is $6100 \mathrm{~Pa} \cdot \mathrm{s}$. The numerical prediction of the steady uniaxial elongational viscosity $\eta_{E}$ using these parameters is shown in Fig.5. The parameters of the WLF equation are $c_{1}=2.94$ and $c_{2}=184.4 \mathrm{~K}$

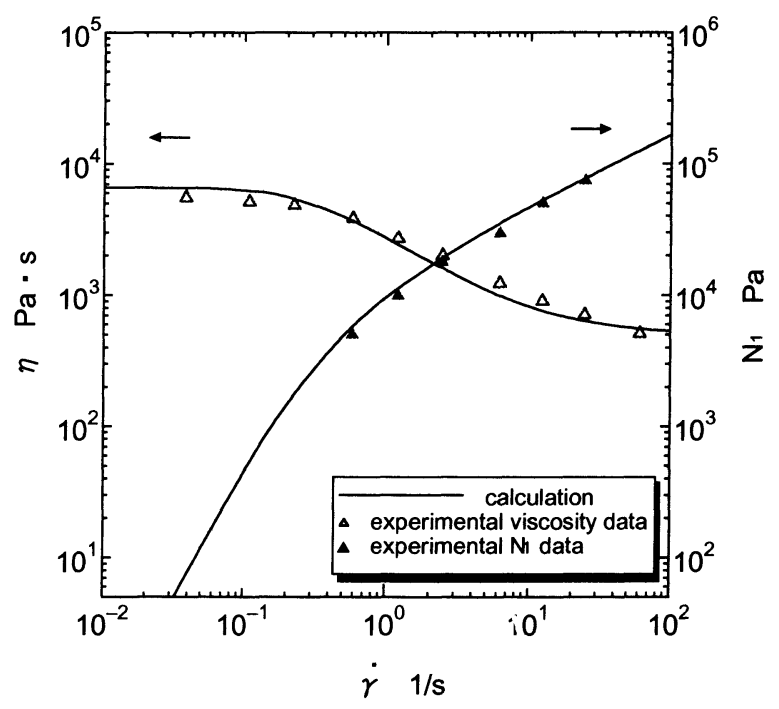

Fig.4 Measurements and predictions using the Giesekus model of steady shear viscosity $\eta$ and the first normal stress difference $N_{1}$ versus shear rate $\gamma$ for LDPE at $190^{\circ} \mathrm{C}$.

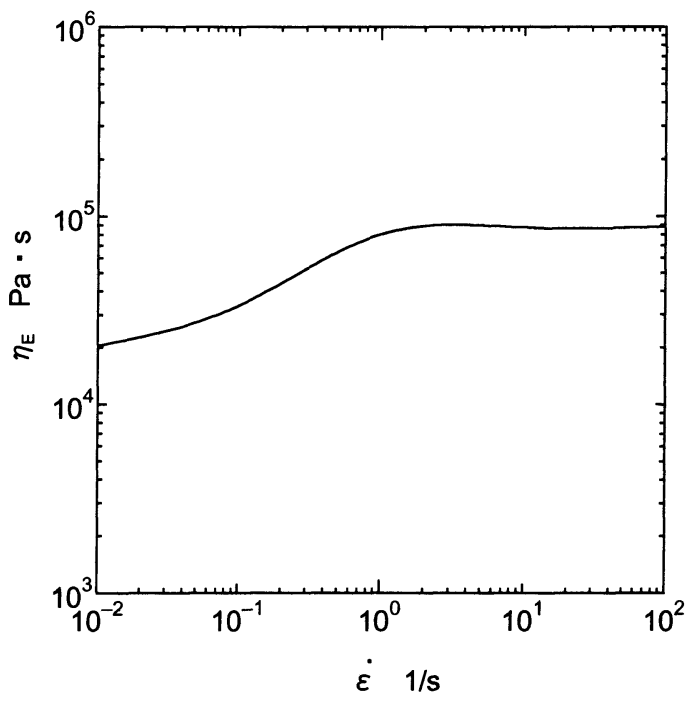

Fig.5 Steady uniaxial elongational viscosity $\eta_{E}$ versus elongational rate $\varepsilon$.

[4]. For other parameters, we aaplied the following values: The density $\rho$ is $762 \mathrm{~kg} / \mathrm{m}^{3}$, the specific heat $C_{p}$ is 2500 $\mathrm{J} /(\mathrm{kg} \cdot \mathrm{K})$, the thermal conductivity $\kappa$ is $0.2 \mathrm{~W} /(\mathrm{m} \mathrm{K})$, and $C=1.18 \times 10^{-9} \mathrm{~Pa}^{-1} \quad[16-18]$.

In Fig. 6 , the schematic diagram of the test channel, the boundary condition, and the coordinate system are indicated. The distance from the spider-rear-end to the channel exit is longer than that in the previous numerical analysis [1] and is the same as that of the visualized region. As the boundary condition of temperature, the following values were applied: The temperature at the inlet $T_{i}$ is $190{ }^{\circ} \mathrm{C}$ and the wall temperature $T_{w}$ is 190 or $205^{\circ} \mathrm{C}$. The spider-front-end angle $\theta_{1}$ and the spider-rear-end angle $\theta_{2}$ were set as $\theta_{1}=45^{\circ}$ $\left(R_{1}=9.7 \mathrm{~mm}\right)$ and $\theta_{2}=45^{\circ}\left(R_{2}=9.7 \mathrm{~mm}\right), 60^{\circ}\left(R_{2}=5.0 \mathrm{~mm}\right)$. The finite element meshes in the computational domain is consists of 3417 nodes and 1600 elements as shown in Fig. 


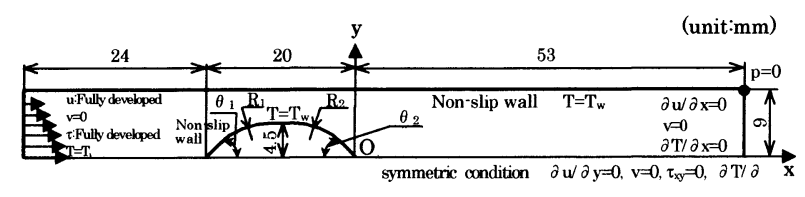

Fig.6 Schematic diagram of a parallel channel with a spider and the boundary conditions.

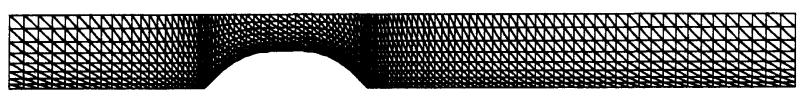

Fig.7 Finite element mesh in the computational domain.

7.

The birefringence measured in the experiment is the average over the channel width. On the other hand, the numerical computation was carried out for two-dimensional flows. Consequently, to compare the numerical results with the experimental measurements, we set the computational condition of mean-entrance-velocities $U_{\text {ent }}$ equal to velocities evaluated by dividing the volumetric flow rates $Q$ by the cross section area under each experimental condition: $U_{\text {ent }}$ $=0.67,0.96,2.1,2.8$, and $4.4 \mathrm{~mm} / \mathrm{s}$.

\section{Results and Discussion}

Figure 8 shows the birefringence patterns at $\theta_{2}=45^{\circ}$ and at the die temperature $T_{d}$ of $190{ }^{\circ} \mathrm{C}$. The patterns were visualized using the photoelastic method. The number of fringes of birefringence increases consistently with the flow rate near the spider and the channel walls, and in the downstream region of the spider rear end. At the maximum flow rate, $Q=4.6 \mathrm{~cm}^{3} / \mathrm{s}$, the increase in the number of fringes is especially large and the birefringence density is high in the region from the spider wall to the merging area. This result indicates that the polymer melt is deformed largely owing to the spider wall and are highly stretched in the flow direction in the merging region at high flow rate.

Next, we investigate the relaxation process of stretched molecules on the centerline. The region where the molecular orientation perfectly relaxes appears black in the photographs in Fig. 8. The birefringence in the weld-line region near the centerline reduces to zero within the visualized region at $Q=0.7,1.0 \mathrm{~cm}^{3} / \mathrm{s}$. At higher flow rates, the orientation does not relax perfectly within the visualized region. The flow birefringence is caused by the molecular orientation in polymer melts [19] hence this experimental result means that the distance need for the relaxation of molecules oriented by the elongation in the merging flow highly depends on the flow rate.

Figure 9 shows the numerical prediction of flow birefringence patterns at $\theta_{2}=45^{\circ}$ and $T_{d}=190^{\circ} \mathrm{C}$, which are described using $\Delta n$ computed according to Eq. (4). In this figure, the number of contours is set as the same as that of fringes in the experiment. The computational pattern agrees well to the experimental counterpart.

In Fig. 10, the experimental data and the computational predictions are compared. The birefringence distributions in the $y z$ section from the spider wall to the end of the

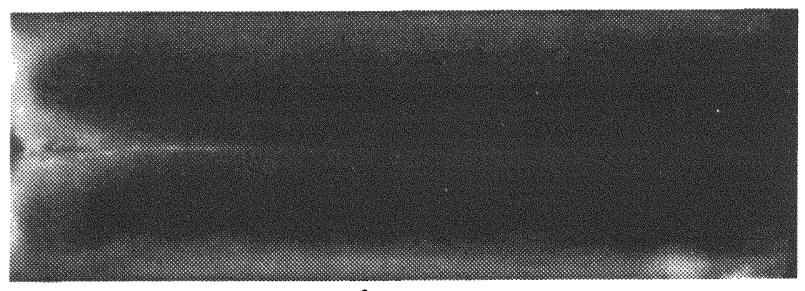

(a) $Q=0.7 \mathrm{~cm}^{3} / \mathrm{s}\left(U_{\text {ent }}=0.67 \mathrm{~mm} / \mathrm{s}\right)$

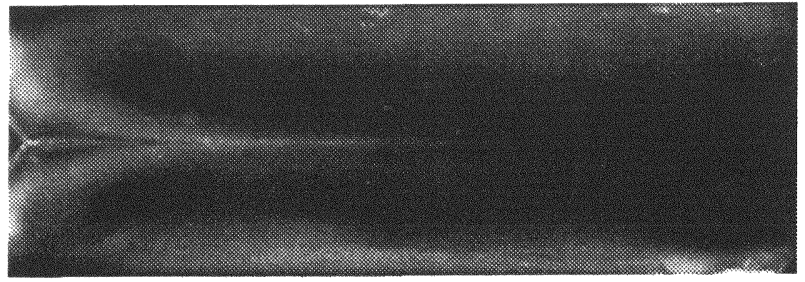

(b) $Q=1.0 \mathrm{~cm}^{3} / \mathrm{s}\left(U_{\mathrm{ent}}=0.96 \mathrm{~mm} / \mathrm{s}\right)$

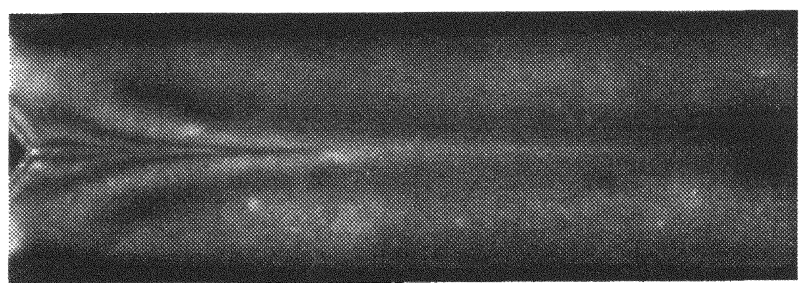

(c) $Q=2.2 \mathrm{~cm}^{3} / \mathrm{s}\left(U_{\mathrm{ent}}=2.1 \mathrm{~mm} / \mathrm{s}\right)$

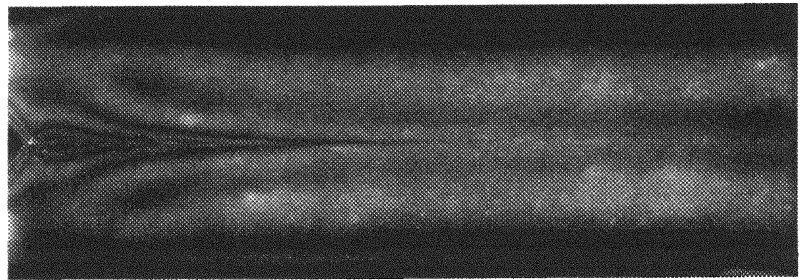

(d) $Q=2.9 \mathrm{~cm}^{3} / \mathrm{s}\left(U_{\text {ent }}=2.8 \mathrm{~mm} / \mathrm{s}\right)$

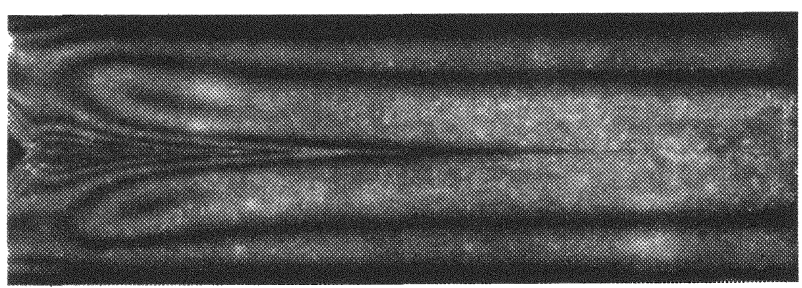

(e) $Q=4.6 \mathrm{~cm}^{3} / \mathrm{s}\left(U_{\text {ent }}=4.4 \mathrm{~mm} / \mathrm{s}\right)$

Fig. 8 Birefringence patterns for the LDPE melt at $\theta_{2}=45^{\circ}$ and $T_{d}=190{ }^{\circ} \mathrm{C}$.

visualized region at $\theta_{2}=45^{\circ}$ are shown. In this figure, the experimental data for $T_{d}=190^{\circ} \mathrm{C}$ and $T_{d}=205^{\circ} \mathrm{C}$ are plotted by open and closed circles, respectively; the numerical predictions for $T_{d}=190^{\circ} \mathrm{C}$ and $T_{d}=205^{\circ} \mathrm{C}$ are expressed by solid and dashed lines, respectively. We considered the experimental data in the half region that corresponds to the computational domain, i.e. $0 \leq \mathrm{y} \leq 9 \mathrm{~mm}$ since the flow birefringence are symmetric with respect to the centerline $(y=0 \mathrm{~mm})$ as shown in Fig.8. In the range from $x=-1.0 \mathrm{~mm}$ to $x=14.1 \mathrm{~mm}$, the birefringence increases towards the channel wall $(y=9 \mathrm{~mm})$, the spider wall, and the centerline $(y=0 \mathrm{~mm})$ under all the conditions of the flow rate and 


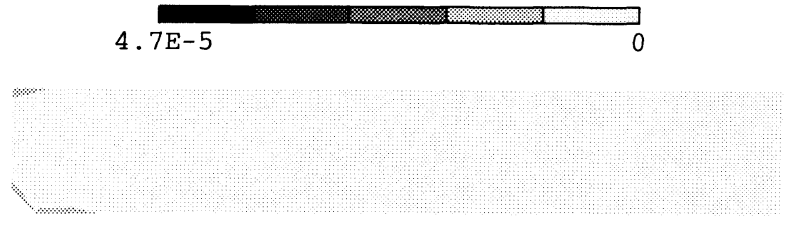

(a) $U_{\text {ent }}=0.67 \mathrm{~mm} / \mathrm{s}$

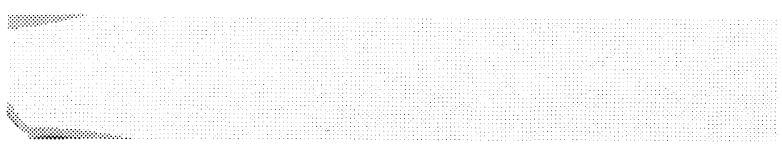

(b) $U_{\text {ent }}=0.96 \mathrm{~mm} / \mathrm{s}$

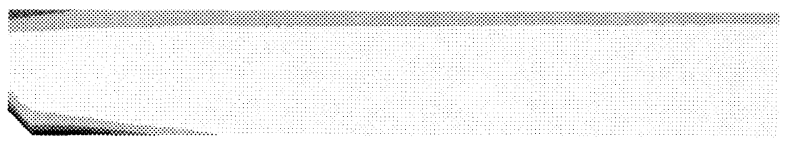

(c) $U_{\text {ent }}=2.1 \mathrm{~mm} / \mathrm{s}$

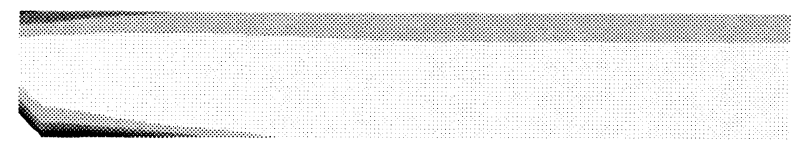

(d) $U_{\text {ent }}=2.8 \mathrm{~mm} / \mathrm{s}$

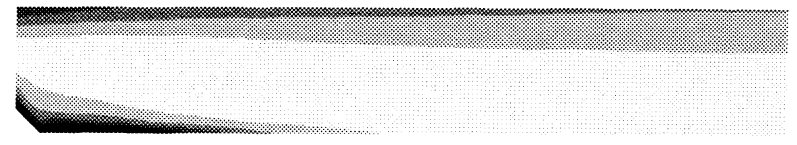

(e) $U_{\text {ent }}=4.4 \mathrm{~mm} / \mathrm{s}$

Fig.9 Birefringence patterns for the calculation at $\theta_{2}=45^{\circ}$ and $T_{w}=190{ }^{\circ} \mathrm{C}$.

temperature. The minimum value is observed near $y=5 \mathrm{~mm}$ in the section of $x=-1.0 \mathrm{~mm}$ and the $y$-position of the minimum birefringence decreases with increasing $x$. At high flow rate, the magnitude of the gradient of $\Delta n$ in the directions towards both $y=0 \mathrm{~mm}$ and $y=9 \mathrm{~mm}$ increases and the maximum value in each section becomes large. Large birefringence is observed on both the spider and channel walls near the section of $x=-1.0 \mathrm{~mm}$. In our previous computation [1], large shear rate on the walls was predicted in this region. Hence it is seen that the birefringence increases because the polymer melt receives large shear deformation near the spider and channel walls. The birefringence in the channel wall side gradually diminishes and relaxes as the melt flows downstream.

At every flow rate and temperature, the magnitude of gradient of the birefringence between $y=0 \mathrm{~mm}$ and $y=5 \mathrm{~mm}$ gradually decreases from $x=-1.0 \mathrm{~mm}$ to $0 \mathrm{~mm}$, conversely, it increases from $x=0 \mathrm{~mm}$ to $x=2.1 \mathrm{~mm}$ and $\Delta n$ grows near $y=0$ $\mathrm{mm}$. The birefringence between $y=0 \mathrm{~mm}$ and $y=5 \mathrm{~mm}$ is the largest at $x=2.1 \mathrm{~mm}$; it decreases downstream of $x=2.1 \mathrm{~mm}$ and the magnitude of its gradient also decreases. The mechanism of this phenomenon is as follows: As indicated in our previous paper [3], the melt separated by a spider encountered shear deformation caused by walls. Before the deformation relaxes enough, the melt is elongated in the flow direction just after the separated flows merge hence the molecular orientation rapidly increases there and decreases in the downstream region. At the maximum flow rate, $Q=4.6$ $\mathrm{cm}^{3} / \mathrm{s}$, the gradient of the birefringence between $y=0 \mathrm{~mm}$ and $y=5 \mathrm{~mm}$ gradually decreases from $x=-1.0 \mathrm{~mm}$ to $0 \mathrm{~mm}$, while it suddenly decreases near $x=26.2 \mathrm{~mm}$. The magnitude of the gradient between $y=0 \mathrm{~mm}$ and $y=5 \mathrm{~mm}$ becomes lower than that caused by the shear deformation between $y=5 \mathrm{~mm}$ and $y=9 \mathrm{~mm}$. This fact means that the stretched molecular orientation relaxes enough if the distance from the spider to the exit is as long as the present experimental apparatus even at high flow rate.

The birefringence is small at $T_{d}=205^{\circ} \mathrm{C}$ compared with that at $190{ }^{\circ} \mathrm{C}$. Because the viscosity of polymer melts decreases with the temperature rise, the stress also decreases and that leads the decrease in birefringence. The difference in viscosity at two temperatures becomes greater with increasing flow rate. Consequently, both the birefringence and the stress decrease remarkably at high temperatures. The effect of the stress relaxation due to the rise of the die temperature appears notably.

The numerical predictions qualitatively agree with the experimental data at $190{ }^{\circ} \mathrm{C}$. Especially at low flow rates, quantitative agreement is also obtained. Quantitative comparison shows that the numerical results are lower than the experimental data in the region of $3 \mathrm{~mm}<y<7 \mathrm{~mm}$ above $Q=2.2 \mathrm{~cm}^{3} / \mathrm{s}$. This is because the predicted shear viscosity of the Giesekus model is lower than the experimental measurement at the shear rate between $1 \mathrm{~s}^{-1}$ and $10 \mathrm{~s}^{-1}$ as shown in Fig. 4.

In the welding region, where the elongational flow is dominant, the numerical prediction of $\Delta n$ and the magnitude of its gradient in the $y$ direction take the maximum in the section of $x=2.1 \mathrm{~mm}$. Both the maximum value and the magnitude of the gradient increase consistently with the flow rate. The numerical predictions of the gradient in the $y$ direction agree with the experimental data. However, the experimental data were not obtained at the position where the computational results take the maximum. This is because the resolution of the measurement device of photoelasticity is not enough to measure the birefringence more precisely near the weld-line on the centerline at high flow rates.

The difference between the numerical results at $T_{w}=190$ and those at $205{ }^{\circ} \mathrm{C}$ becomes large when the flow rate is large. However, the difference is small as compared with the experimental results. Moreover, the numerical prediction at $y=9 \mathrm{~mm}$ near the channel wall is larger at $T_{w}=205^{\circ} \mathrm{C}$ than at $T_{w}=190^{\circ} \mathrm{C}$; this result is contrary to the experiment. In our previous numerical analysis of the two-dimensional flow [4], local temperature rise near walls leads the increase in the velocity gradient in this area and hence the stress also increases. In the present experiments, however, the local temperature distribution in the $y$ direction did not occur because the effect of the local temperature rise was reduced by both the heat transfer in the $z$ direction from the channel wall to the fluid and the remaining heat on the pipe coupling of the die and the adaptor. Hence the viscosity became low and the birefringence decreases almost uniformly in the $y$ direction. 
Next, we analyzed the results for the channel with the spider of $\theta_{2}=60^{\circ}$. Figure 11 shows the birefringence distribution in the $y$ direction in each $y z$ section from the spider wall to the exit of the visualized region. In the experiment, the growth of the magnitude of birefringence gradient towards both the spider wall and channel wall at $y=9 \mathrm{~mm}$ is greater than the results at $\theta_{2}=45^{\circ}$. This tendency is outstanding in the direction to the spider wall since the $x=-1.0 \quad 0$
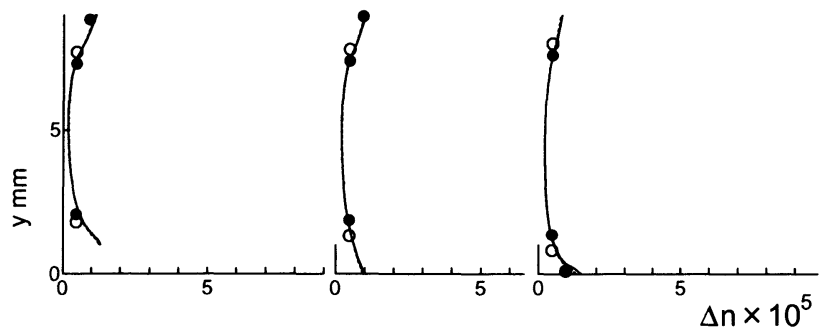

(a) $Q=0.7 \mathrm{~cm}^{3} / \mathrm{s}$
4.2

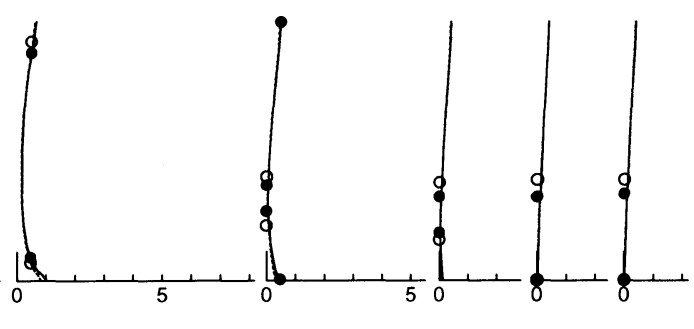

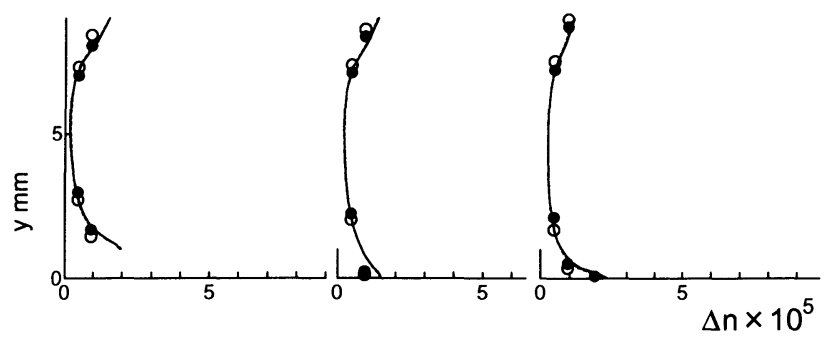

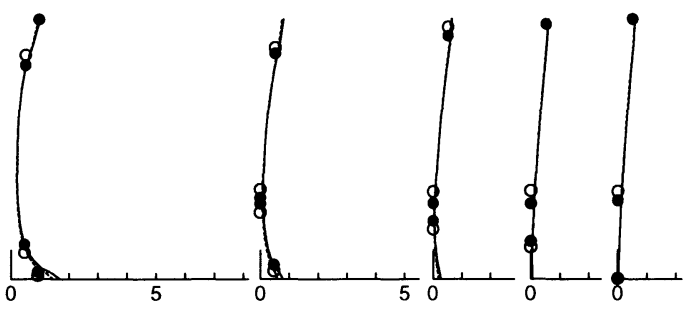

(b) $Q=1.0 \mathrm{~cm}^{3} / \mathrm{s}$

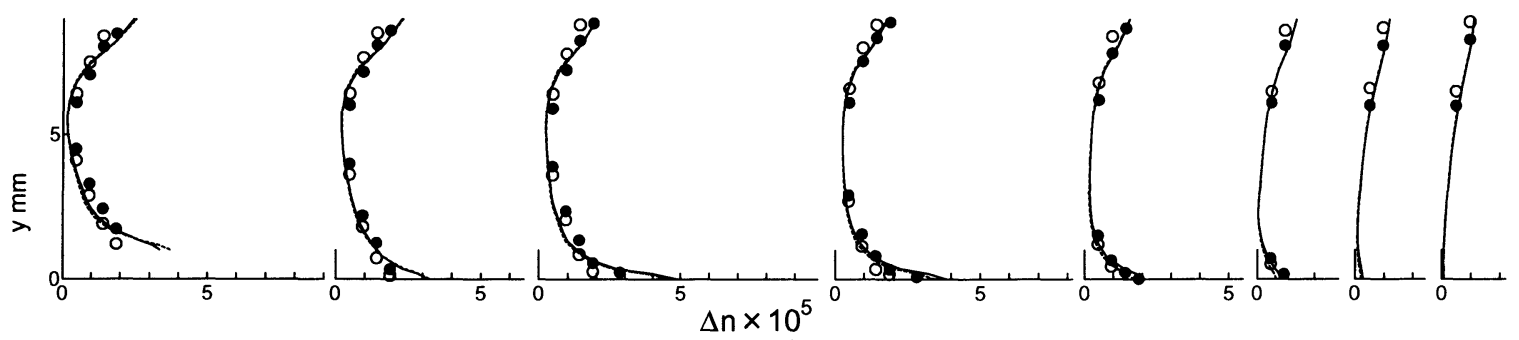

(c) $Q=2.2 \mathrm{~cm}^{3} / \mathrm{s}$
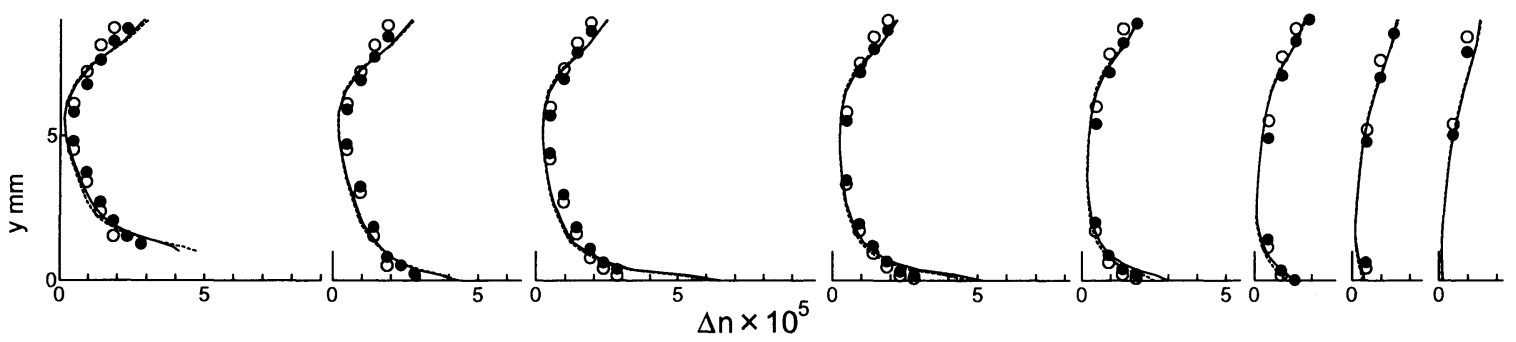

(d) $Q=2.9 \mathrm{~cm}^{3} / \mathrm{s}$

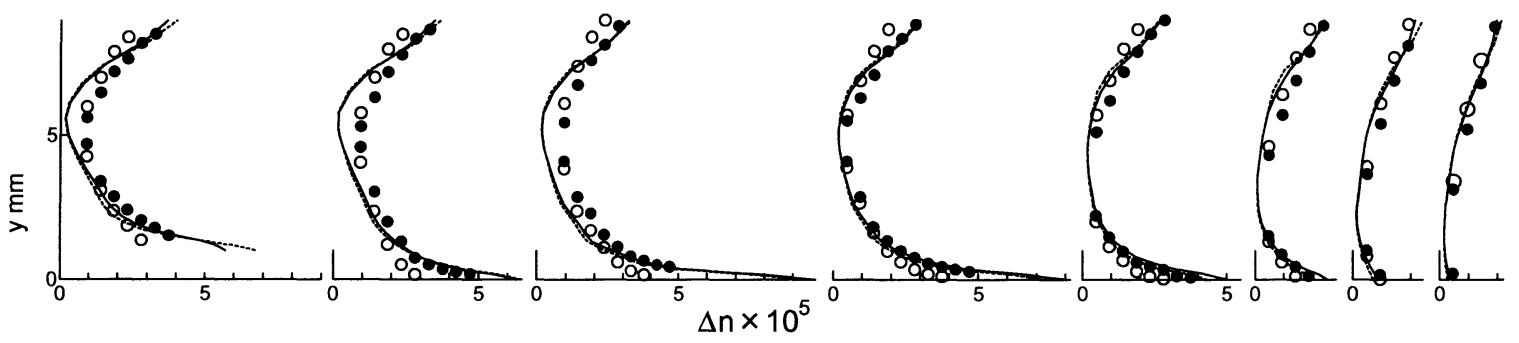

(e) $Q=4.6 \mathrm{~cm}^{3} / \mathrm{s}$

Fig.10 Birefringence distribution at different cross sections and $\theta_{2}=45^{\circ}$ for the LDPE melt. Symbols: experiment $\left(\mathrm{O}^{\circ} T_{d}=190{ }^{\circ} \mathrm{C}\right.$,

$\left.\bigcirc: T_{d}=205{ }^{\circ} \mathrm{C}\right)$; curves: calculation (

$: T_{w}=190{ }^{\circ} \mathrm{C}$,

: $\left.T_{w}=205{ }^{\circ} \mathrm{C}\right)$. 
shear stress caused by the shear deformation near the spider and channel walls is larger than that at $\theta_{2}=45^{\circ}$. In this region where expanding flows occur, slope of the spider wall is large and the channel width is narrow in this section in the case of $\theta_{2}=60^{\circ}$. Consequently, the fluid is sheared more strongly than at $\theta_{2}=45^{\circ}$ and hence the gradient of the birefringence in the $y$ direction increases. At the maximum flow rate, $Q=4.6 \mathrm{~cm}^{3} / \mathrm{s}$, the increase in the gradient in the $y$

$$
x=-1.0
$$
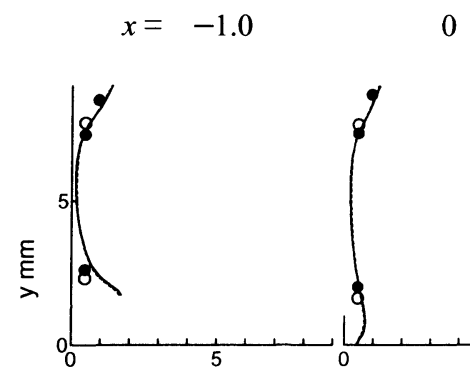

2.1

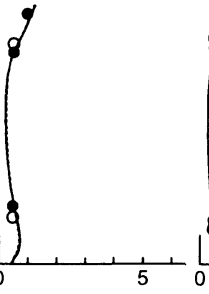

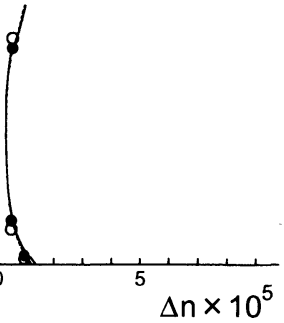

4.2

\section{$\begin{array}{llll}8.2 & 14.1 \quad 26.2 \quad 46.0 \mathrm{~mm}\end{array}$}

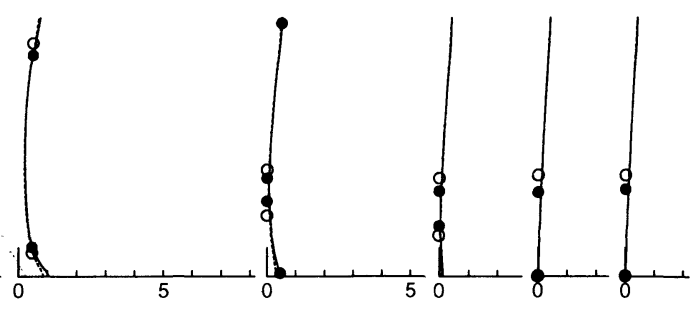

(a) $Q=0.7 \mathrm{~cm}^{3} / \mathrm{s}$
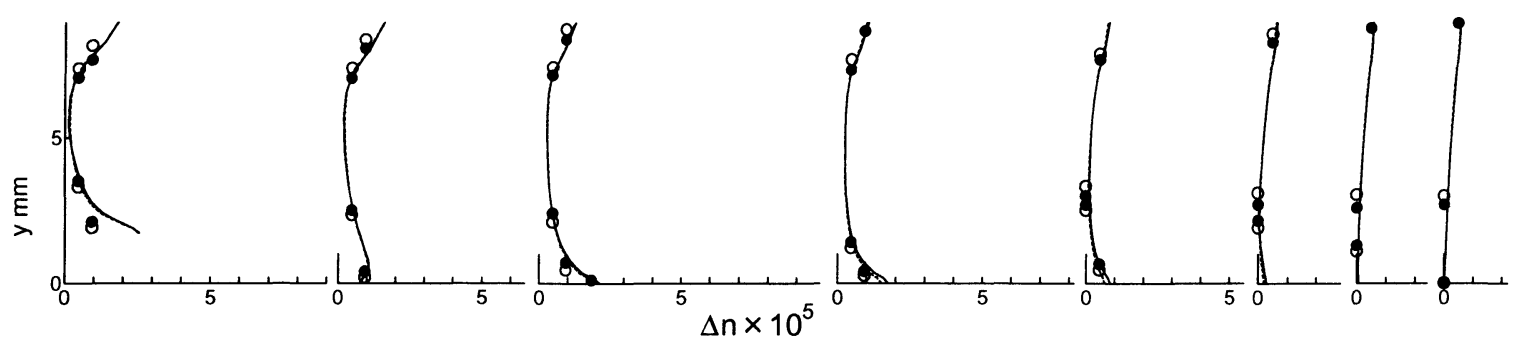

(b) $Q=1.0 \mathrm{~cm}^{3} / \mathrm{s}$

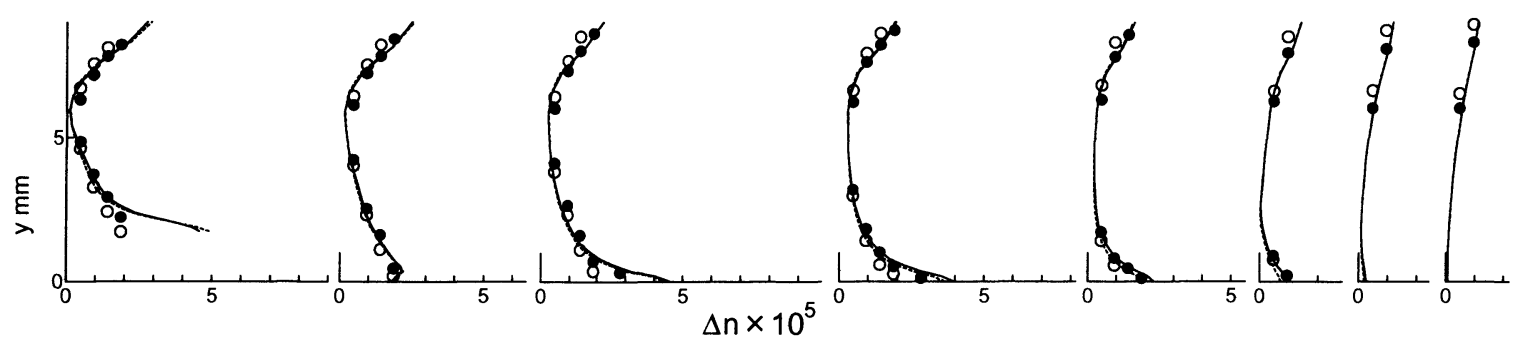

(c) $Q=2.2 \mathrm{~cm}^{3} / \mathrm{s}$

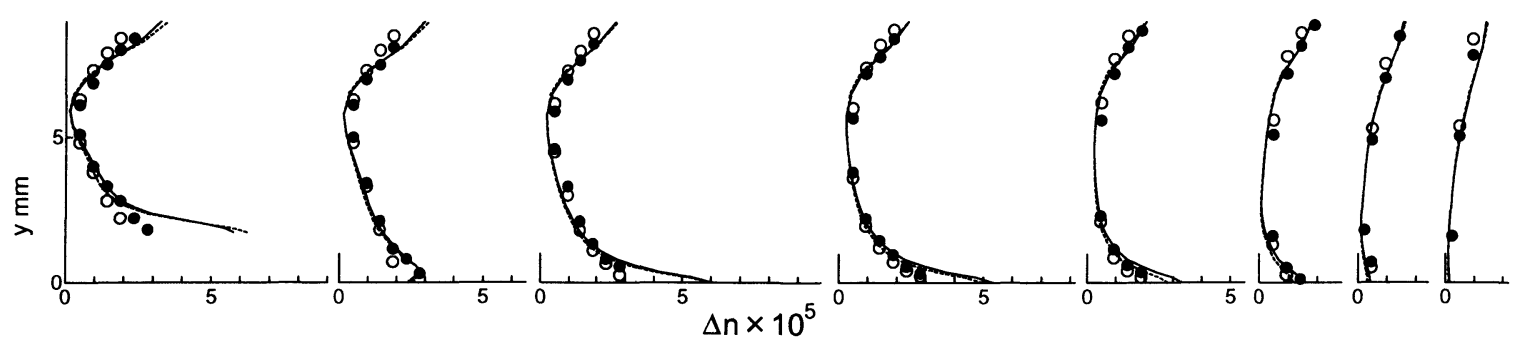

(d) $Q=2.9 \mathrm{~cm}^{3} / \mathrm{s}$
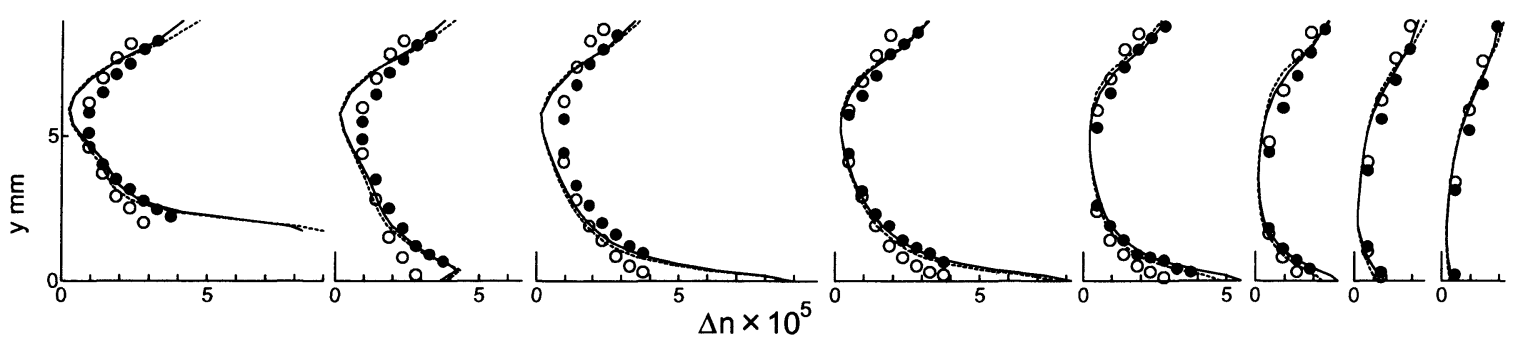

(e) $Q=4.6 \mathrm{~cm}^{3} / \mathrm{s}$

Fig. 11 Birefringence distribution at different cross sections and $\theta_{2}=60^{\circ}$ for the LDPE melt. Symbols: experiment

$T_{d}=190{ }^{\circ} \mathrm{C}$ O: $T_{d}=205{ }^{\circ} \mathrm{C}$ ); curves: calculation $\left(-: T_{w}=190{ }^{\circ} \mathrm{C}\right.$, - : $T_{w}=205{ }^{\circ} \mathrm{C}$ ). 
direction near the spider and channel walls occurs in each cross sections downstream of the merging region, gradually decreases towards the exit, and almost vanished at $x=14 \mathrm{~mm}$. The birefringence gradient vanishes at smaller $x$ position at lower flow rate.

In the case of $\theta_{2}=60^{\circ}$, the numerical results also agree well with the experimental measurements. In the section of $x=0$ $\mathrm{mm}$, there are differences between the results at $\theta_{2}=45^{\circ}$ and those at $\theta_{2}=60^{\circ}$ in the birefringence distributions in the welding region near $y=0 \mathrm{~mm}$. The birefringence at $x=0 \mathrm{~mm}$ increases towards the centerline but becomes low near the centerline at $\theta_{2}=60^{\circ}$, while the birefringence takes the maximum at $y=0 \mathrm{~mm}$ at $\theta_{2}=45^{\circ}$. This is because the maximum value at $\theta_{2}=60^{\circ}$ is small in comparison with that at $\theta_{2}=45^{\circ}$ as shown in the previous paper [3]. This phenomenon was not observed in the experiment because of low resolution of the measurement device.

Figures 12 and 13 show the distributions of the birefringence along the centerline in the visualized region downstream of the spider rear end at $\theta_{2}=45^{\circ}, 60^{\circ}$, respectively. In both the experimental and numerical results, the maximum birefringence becomes greater and the distance need for its relaxation extends with increasing flow rate. On the centerline, there is no shear deformation theoretically, thus the birefringence is caused only by the elongational stress. Consequently, we can understand that the elongational stress grows in the weld-line region where the elongational flow is dominant and gradually decreases in the downstream region. The growth of the elongational stress becomes greater and the distance for its relaxation becomes longer with increasing flow rate. If the distance from the spider rear end to the die exit is fixed, it depends on the flow rate whether the molecular orientation returns to a random orientation; Anisotropy remains in products when the flow rate is large.

In the experiment, the birefringence at every flow rate is small at $T_{d}=205^{\circ} \mathrm{C}$ as compared with the results at $T_{d}=190$ ${ }^{\circ} \mathrm{C}$ since the elongational viscosity decreases owing to the temperature rise. The difference between the results at two temperatures appears remarkably at high flow rate. Hence, it is efficient to raise the die temperature for the restriction of both the growth of elongational stress and the extension of the relaxation distance caused by the increase in the flow rate.

The results for $\theta_{2}=45^{\circ}$ show similar tendency to those for $\theta_{2}=60^{\circ}$ : Both the distance need for the relaxation and the birefringence at $x=53 \mathrm{~mm}$ for $\theta_{2}=45^{\circ}$ are almost the same as counterparts at $\theta_{2}=60^{\circ}$. That is, the spider shape does not affect the relaxation of molecular orientation on the weld-line if the distance for the relaxation is long enough.

Good agreement between the computation and the experimental data indicates that the numerical prediction of molecular orientation in the weld-line region is appropriate. Although the numerical computation considered two-dimensional flows, it predicts the experimental results in three-dimensional flows well. This is because the channel height is enough larger than the channel width and hence the flow can be approximated to be two-dimensional; in addition,

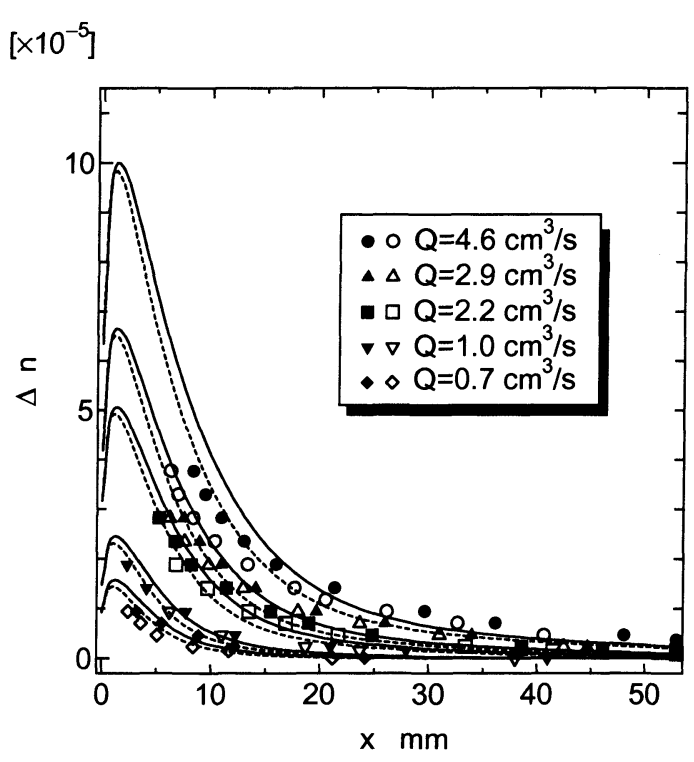

Fig.12 Birefringence along the center line downstream of the spider rear end at $\theta_{2}=45^{\circ}$ for the LDPE melt. Symbols: experiment $\left(\boldsymbol{O}, \boldsymbol{\Delta}, \boldsymbol{\square}, \boldsymbol{\nabla}, \diamond: T_{d}=190^{\circ} \mathrm{C}, \bigcirc\right.$, $\triangle, \square, \nabla, \diamond: T_{d}=205{ }^{\circ} \mathrm{C}$ ); curves: calculation ( - : $T_{w}=190{ }^{\circ} \mathrm{C}$, ---- : $T_{w}=205{ }^{\circ} \mathrm{C}$ ).

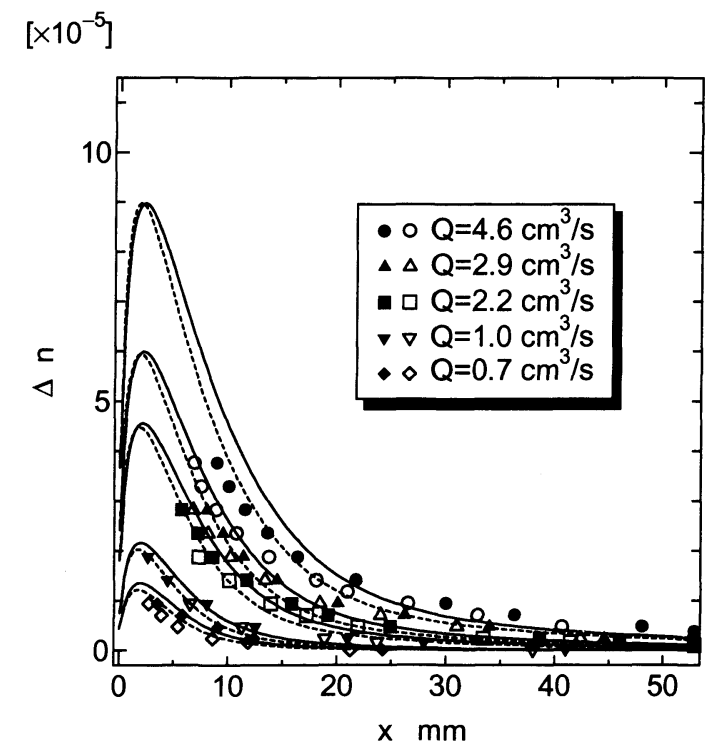

Fig.13 Birefringence along the center line downstream of the spider rear end at $\theta_{2}=60^{\circ}$ for the LDPE melt. Symbols: experiment $\left(\boldsymbol{O}, \boldsymbol{\Lambda}, \boldsymbol{\square}, \boldsymbol{\nabla}, \diamond: T_{d}=190^{\circ} \mathrm{C}, \mathrm{O}\right.$, $\triangle, \square, \nabla, \diamond: T_{d}=205{ }^{\circ} \mathrm{C}$ ); curves: calculation ( - : $T_{w}=190{ }^{\circ} \mathrm{C},-\cdots-: T_{w}=205^{\circ} \mathrm{C}$ ).

the average birefringence over the width calculated by Eq. (2) was used as the experimental data. The effect of large $\theta_{2}$ on the restriction of the maximum elongational stress which is indicated in our previous study [1] was also shown in the present numerical prediction. However, this effect was not confirmed in the experiment because of low resolution of the measurement device. For fluids with stretch-thickening 
elongational viscosity, the restriction effect of large $\theta_{2}$ on the relaxation of molecular orientation was not strong and the effect of temperature rise was more effective as shown in the previous paper [1]. In addition, the three-dimensional heat transfer probably caused the temperature rise of polymer melt and progressed the relaxation of molecular orientation.

\section{Conclusion}

We carried out the flow birefringence measurement in welding flows caused by a spider using a LDPE melt as a test fluid. We investigated the effects of rheological properties of the melt, the spider shape, the flow rate, and the die temperature on the molecular orientation that appear in the birefringence in the welding region. The experimental results were compared to the numerical prediction of non-isothermal viscoelastic welding flow using the single mode Giesekus model.

On the centerline where the elongational flow is dominant, the growth of elongational stress and its relaxation distance is affected by the flow rate. Consequently, it depends on the flow rate whether the orientation relaxes to a perfect random state or not. If the distance to the exit is not long enough, anisotropy remains in products when the flow rate is large. The relaxation of the orientation of stretched molecules, however, progresses even at high flow rate.

When the die temperature is higher than the melt temperature, the growth in elongational stress due to the increase in the flow rate is restricted and the distance for stress relaxation becomes short. As a result, the anisotropy in products decreases.

When the distance for the relaxation of molecular orientation is long enough, the restriction of the maximum elongational stress using a spider with a large $\theta_{2}$ does not affect to the relaxation of molecular orientation in the weld-line region for a strong stretch-thickening fluid, and the rise in the die temperature shows great effect to the relaxation. These results are consistent with the previous prediction [1]. In addition, the numerical prediction agrees well with the experimental data under all conditions of the flow rate and the temperature.

\section{References}

[1] Mitsuhashi, M., Nishimura, K., Nomura, K., Yamamoto, T., Mori, N. and Nakamura, K.; J. Text. Eng., 47, 1 (2001)

[2] Giesekus, H.; J. Non-Newtonian Fluid Mech., 11, 69 (1982)

[3] Huang, Y. and Prentice, P.; Polym. Eng. Sci., 38, 1506 (1998)

[4] Mitsuhashi, M., Nishimura, K., Nomura, K., Yamamoto, T., Mori, N. and Nakamura, K.; J. Text. Mach. Soc. Japan, 54, T149 (2001)

[5] Isayev, A. I.; J. Non-Newtonian Fluid Mech., 19, 135 (1985)

[6] Kiriakidis, D. G., Park, H. J. and Mitsoulis, E.; J. Non-Newtonian Fluid Mech., 47, 339 (1993)

[7] Piau, J.-M., Kissi, N. and Mezghani, A.; J. Non-Newtonian Fluid Mech., 59, 11 (1995)

[8] Li, J.-M., Burghardt, W. R., Yang, B. and Khomami, B.; J. Non-Newtonian Fluid Mech., 74, 151 (1998)

[9] Venerus, D. C., Zhu, S.-H. and Öttinger, H. C.; J. Rheol., 43, 795 (1999)

[10] Baaijens, F. P. T., Selen, S. H. A., Baaijens, H. P. W., Peters, G.W.M. and Meijer, H. E. H.; J. Non-Newtonian Fluid Mech., 68, 173 (1997)

[11] Wei, K. H., Nordberg III, M. E. and Winter, H. H.; Polym. Eng. Sci., 27, 1390 (1987)

[12] Saito, T., Satoh, I., Uesugi, K. and Handa, K.; Seikei-Kakou, 12, 325 (2000)

[13] Philippoff, W.; J. Appl. Phys., 27, 984 (1956)

[14] Philippoff, W.; Trans. Soc. Rheol., 5, 163 (1961)

[15] Philippoff, W. and Gill, S. J.; Trans. Soc. Rheol., 7, 33 (1963)

[16] Horikawa, A., Nakamura, K. and Umegaki, S.; J. Text. Mach. Soc. Japan, 29, T63 (1976)

[17] Nakamura, K., Ishizaki, K., Yamamoto, Y., Amazutsumi, T. and Horikawa, A.; J. Text. Mach. Soc. Japan, 30, T104 (1976)

[18] Tanifuji, S., Kikuchi, T., Takimoto, J. and Koyama, K.; Polym. Eng. Sci., 40, 1878 (2000)

[19] Kikutani, T. and Itou, H.; Seikei-Kakou, 12, 427 (2000) 